\title{
Imaging Findings in Auto-Atticotomy
}

\author{
M. Manasawala, M.E. Cunnane, H.D. Curtin, and G. Moonis
}

\begin{abstract}
BACKGROUND AND PURPOSE: An acquired attic cholesteatoma may spontaneously drain externally into the external auditory canal, leaving a cavity in the attic with the shape of the original cholesteatoma but now filled with air, a phenomenon referred to as "nature's atticotomy" or auto-atticotomy. We describe and quantify the CT appearance of the auto-atticotomy cavity as it pertains to the appearance of the scutum and the lateral attic wall.
\end{abstract}

MATERIALS AND METHODS: Twenty-one patients with erosion of the scutum and loss of the lower attic wall on MDCT were identified during a 5-year span. Images were assessed for measureable widening of the space between the ossicles and the lower lateral attic wall in the axial and coronal planes. Three measurements of the lateral attic were made on the axial images. Findings were compared with the same measurements in 20 control subjects.

RESULTS: The 21 patients had a characteristic blunting of the scutum with loss of the lower lateral attic wall and widening of the lateral attic, consistent with an auto-atticotomy. There was a statistically significant $(P<.001)$ widening of the lateral attic dimensions in the axial plane in the patients with auto-atticotomy.

CONCLUSIONS: Spontaneously evacuated cholesteatoma may mimic a surgical atticotomy on MDCT. Scutal erosion and attic enlargement with a smoothly contoured bony remodeling of the lower lateral attic wall in a patient with no history of surgery suggest that a cholesteatoma was previously present and spontaneously drained.

A $\mathrm{n}$ atticotomy is a surgical approach through the external auditory canal to the attic of the middle ear, whereby the scutum and the lower lateral wall of the attic are surgically removed for access. An auto-atticotomy (also called "nature's atticotomy") refers to an enlarged lateral attic with absence of the scutum and lower lateral wall of the attic in a patient without a history of surgery. ${ }^{1-3}$ This entity results from a deep retraction pocket or a cholesteatoma that has eroded the bone and then spontaneously drained into the external auditory canal. The wall of the original cholesteatoma remains, and so the air-filled defect is lined by keratinizing squamous epithelium. The appearance of an atticotomy and an auto-atticotomy is similar on CT. The purpose of this article was to describe the findings of auto-atticotomy on multidetector CT of the temporal bone.

Received January 18, 2013; accepted after revision March 22.

From the Department of Radiology (M.M.), Abington Memorial Hospital, Abington, Pennsylvania; Department of Radiology (G.M.), Beth Israel Deaconess Medical Center, Boston, Massachusetts; and Department of Radiology (M.E.C., H.D.C., G.M.), Massachusetts Eye and Ear Infirmary, Boston, Massachusetts.

Please address correspondence to Gul Moonis, MD, Department of Radiology, Massachusetts Eye and Ear Infirmary, 243 Charles St, Boston, MA 02215; e-mail: gmoonis@bidmc.harvard.edu

http://dx.doi.org/10.3174/ajnr.A3791

\section{MATERIALS AND METHODS}

The study was approved by the Human Studies Committee of our hospital, and review of on-line medical records was performed in all patients and controls. The images were prospectively collected by 2 of the authors (practicing head and neck radiologists) during a 5-year period from MDCT scans of the temporal bone viewed in daily readouts. The inclusion criteria were the following: 1) apparent absence or blunting of the scutum and/or smooth bony remodeling of the lateral attic wall on MDCT, and 2) no history of prior otologic surgical intervention. This yielded 21 patients ( 12 men and 9 women, 27-88 years of age) who fulfilled the inclusion criteria; additionally, all had a history of chronic otitis media. Twelve patients had confirmation of pars flaccida cholesteatoma by histology, 2 patients had evidence of pars flaccida cholesteatoma on clinical examination, and 8 patients had deep attic retraction pockets on clinical examination. These 8 patients all had history of intermittently draining ears. We also retrospectively evaluated MDCT of the temporal bone in 20 control subjects. These control subjects had been referred to the radiology department for unrelated symptoms such as tinnitus or sensorineural hearing loss and did not have any history of prior 

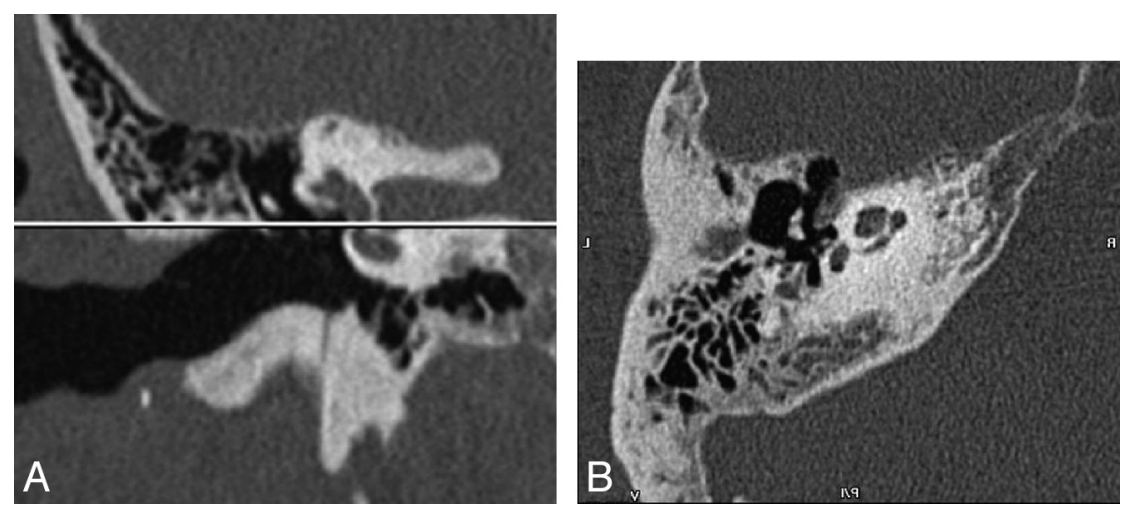

FIG 1. A, Coronal MDCT image depicts the location of the axial plane at the base of the scutum. $B$, Axial MDCT image at the level where the first bone image appears superior to the aircontaining external auditory canal. The malleoincudal junction is well-seen.
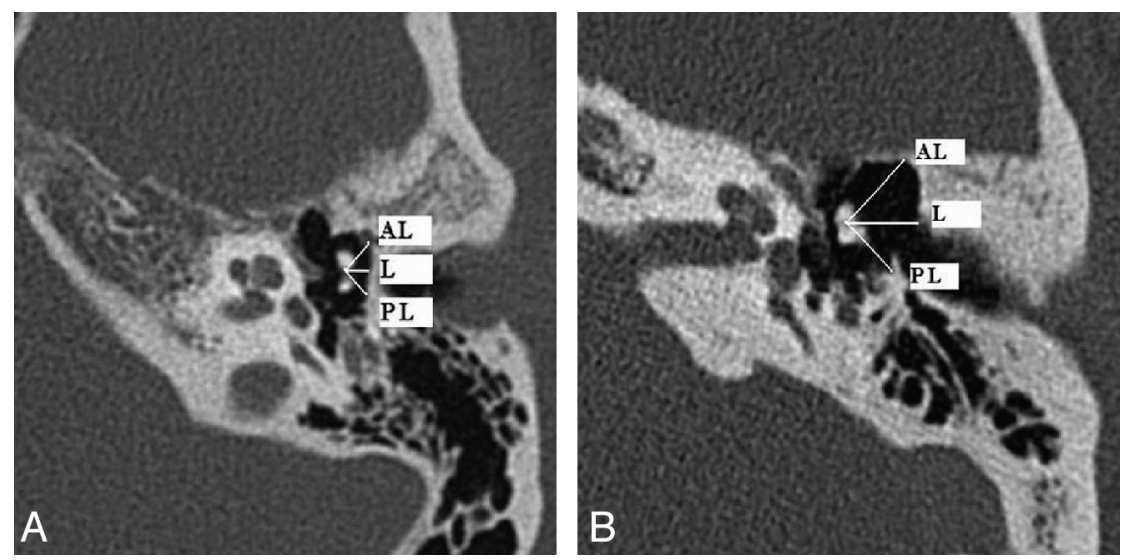

FIG 2. A, Lateral attic measurements in a healthy subject. Axial MDCT demonstrates anterolateral $(\mathrm{AL})$, lateral $(\mathrm{L})$, and posterolateral $(\mathrm{PL})$ measurements from the center of the malleoincudal articulation at the level of the base of the scutum in a healthy subject. $B$, Lateral attic measurements in a 27-year-old patient with a history of cholesteatoma. There is a left auto-atticotomy with smooth remodeling and widening of the anterolateral (AL), lateral $(\mathrm{L})$, and posterolateral $(\mathrm{PL})$ walls of the attic.

Table 1: Lateral, anterolateral, and posterolateral attic measurements $(\mathrm{mm})$ in patients

\begin{tabular}{cccc}
\hline Patients & Lateral & Anterolateral & Posterolateral \\
\hline A & 3.6 & 4.6 & 3.8 \\
B & 3.8 & 4.5 & 3.7 \\
C & 5 & 4.4 & 3.2 \\
D & 3.6 & 5 & 3.3 \\
E & 3.2 & 4 & 2.5 \\
F & 4.3 & 3.8 & 3.7 \\
G & 4.3 & 4.1 & 4.5 \\
H & 2.3 & 3.3 & 2.3 \\
I & 4.8 & 4.4 & 6.9 \\
J & 3.7 & 3.6 & 4.3 \\
K & 3.9 & 4.1 & 4.1 \\
L & 3.7 & 3.6 & 3.9 \\
M & 7.5 & 4.3 & 4.5 \\
N & 3.7 & 4.2 & 3.5 \\
O & 4.7 & 5.5 & 3.9 \\
P & 3.9 & 4 & 3.4 \\
Q & 5.4 & 7.4 & 4.2 \\
R & 3.5 & 3.6 & 4.7 \\
S & 3.5 & 4.8 & 3.3 \\
T & 3.8 & 5.2 & 4.4 \\
U & 4.1 & 4.2 & 3.8 \\
\hline & & &
\end{tabular}

middle ear inflammatory disease or surgery of the temporal bone; their tympanic membranes were normal at otoscopy.

All subjects had imaging performed on a 40 -section multidetector CT scanner (Somatom Sensation scanner; Siemens, Erlangen, Germany). The raw data were acquired helically with $0.6-\mathrm{mm}$ collimation and 0.55 pitch at $320 \mathrm{mAs}$ and 120 $\mathrm{kV}$ (peak), with the scan excursion plotted from the arcuate eminence through the mastoid tip. The raw data from each ear were separated and reconstructed into 0.6 (section thickness) $\times 0.2 \mathrm{~mm}$ (reconstruction interval) axial images in bone algorithms at a display FOV of $100 \mathrm{~mm}$ and a matrix of $512 \times 512$. The data were displayed in 3 orthogonal planes on the scanner console. The technologist scrolled through the sagittal data to find an image on which the anterior and posterior limbs of the lateral semicircular canal were displayed in cross-section. An axial dataset was made in a standardized plane parallel to the lateral semicircular canal by placing the reference line connecting the anterior and posterior limbs of the canal. Axial images were reformatted at 0.6 (image thickness $) \times 0.5$ (distance between images) $\mathrm{mm}$. Coronal images $(0.6 \times 0.5 \mathrm{~mm})$ were made in a plane perpendicular to the axial images.

The images were visually inspected for scutal erosion, widening of the lateral attic, middle ear opacification, ossicular erosion, and mastoid opacification. To quantify our subjective visual impression of the auto-atticotomy changes, we performed the following measurements: An axial image was chosen that demonstrated the malleoincudal junction at the level of the base of the scutum. For this purpose, the base of the scutum was determined to be the first bone image superior to the air-containing external auditory canal and was reproducibly seen in all cases (Fig $1 A,-B)$. Three measurements were obtained for each case. A lateral measurement was made from the center of the malleoincudal junction to the base of the scutum, and subsequent measurements were made at a $45^{\circ}$ angle anterior and a $45^{\circ}$ angle posterior to this initial measurement (Fig 2A, $-B$ and Tables 1 and 2).

We performed statistical analysis by using the Statistical Package for the Social Sciences, Version 13.0 software (IBM, Armonk, New York). We used the Mann-Whitney $U$ test, a nonparametric test to assess whether there was a statistically significant difference between the 2 samples of observations in patients and controls.

\section{RESULTS}

The lateral attic dimension measured an average of $4.11 \pm 1.05$ $\mathrm{mm}$ in patients compared with $2.37 \pm 0.37 \mathrm{~mm}$ in healthy sub- 
Table 2: Lateral, anterolateral, and posterolateral attic measurements $(\mathrm{mm})$ in controls

\begin{tabular}{cccc}
\hline Controls & Lateral & Anterolateral & Posterolateral \\
\hline AA & 2.8 & 2.7 & 2.7 \\
BB & 3 & 3.4 & 3 \\
CC & 2.4 & 3 & 2.6 \\
DD & 1.9 & 3.6 & 2.2 \\
EE & 2 & 3.1 & 2.3 \\
FF & 1.8 & 2.4 & 2 \\
GG & 1.8 & 2.6 & 1.9 \\
HH & 2.5 & 4.2 & 2.3 \\
II & 2.3 & 3.8 & 2.6 \\
J & 2.3 & 3.2 & 2.5 \\
KK & 2.9 & 3.6 & 2.3 \\
LL & 2.1 & 3.2 & 2.3 \\
MM & 2.2 & 2.3 & 2.4 \\
NN & 2.3 & 3.1 & 2.3 \\
OO & 2.5 & 2.7 & 2.2 \\
PP & 2.9 & 3.8 & 2.9 \\
QQ & 2.1 & 2.6 & 2.4 \\
RR & 2.3 & 2.4 & 2.3 \\
SS & 2.6 & 3.6 & 3.3 \\
TT & 2.8 & 3.6 & 3.2 \\
& & &
\end{tabular}

jects. These results represented a significant statistical difference between the 2 groups with a 2-tailed $P$ value of $<.001$.

The anterolateral attic dimension measured an average of $4.41 \pm 0.88$ in patients compared with $3.15 \pm 0.54 \mathrm{~mm}$ in healthy subjects. These results represented a significant statistical difference between the 2 groups with a 2 -tailed $P$ value of $<.001$.

The posterolateral attic dimension measured an average of $3.90 \pm 0.93 \mathrm{~mm}$ in patients compared with $2.49 \pm 0.37 \mathrm{~mm}$ in healthy subjects. These results represented a significant statistical difference between the 2 groups with a 2 -tailed $P$ value of $<.001$.

Overall, $12 / 21$ patients (57\%) demonstrated minimal or no soft-tissue opacification within the middle ear cavity. Nine patients had nonspecific middle ear opacification. Mastoid underpneumatization and/or opacification was seen in 17/21 (80\%) patients. Ossicular erosion was seen in 10/21 (47\%) patients.

\section{DISCUSSION}

Pars flaccida or primary acquired cholesteatoma most commonly forms in the Prussak space lateral to the ossicles and may result in medial ossicular displacement and/or erosion. Lateral expansion causes erosion of the scutum and lower lateral wall of the attic. ${ }^{4-7}$ Histologically, cholesteatoma is a cystic structure containing exfoliated keratin lined by stratified keratinizing squamous epithelium. The outer layer of the cyst lining or sac is composed of perimatrix or lamina propria. ${ }^{8}$ There are multiple theories to the explain the pathogenesis of bony destruction associated with cholesteatomas, including remodeling from local pressure and recruitment of osteoclasts that are enzymatically active. ${ }^{9}$

At imaging (usually CT), a cholesteatoma classically is seen as a soft-tissue mass adjacent to the ossicles with erosion of the ossicles or the scutum. If the cholesteatoma drains spontaneously into the external auditory canal, the bony changes remain but the mass is no longer visualized. Instead, the "cavity" is now filled with air.

An auto-mastoidectomy has been described referring to external evacuation of the contents of a cholesteatoma that had replaced much of the mastoid, leaving behind its outer mem-

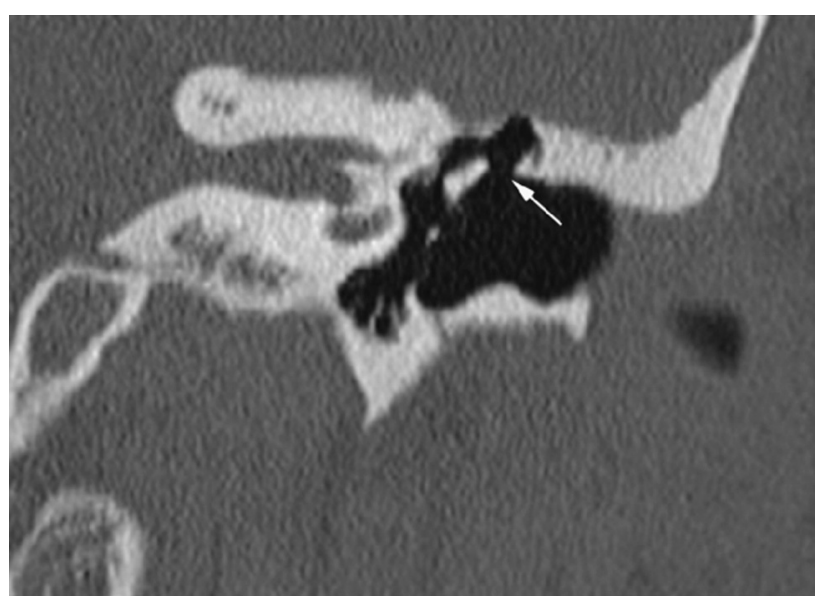

FIG 3. Auto-atticotomy in a 35-year-old patient with a remote history of draining ear. Coronal MDCT demonstrates scutal absence (arrow) mimicking the appearance of a surgical atticotomy. Note the lack of soft-tissue inflammatory changes.

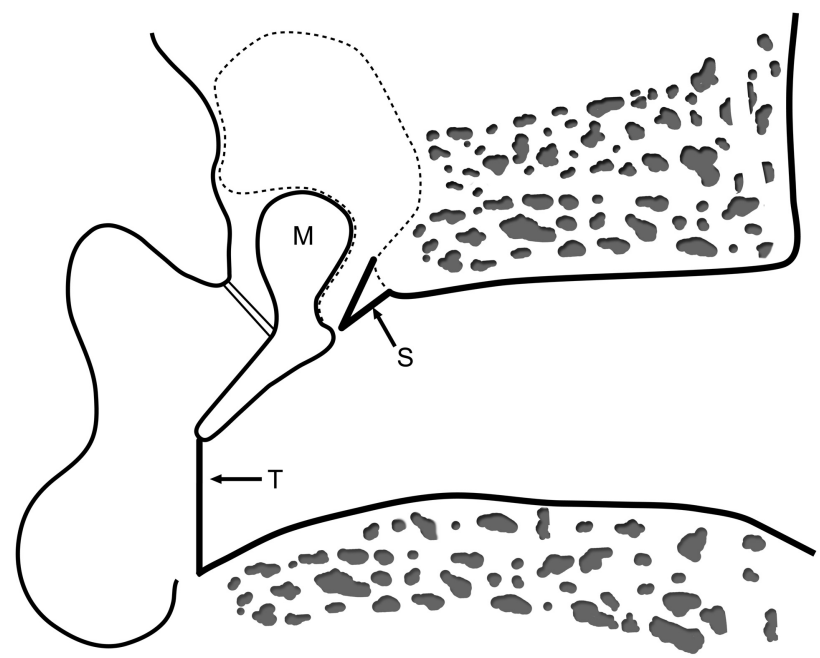

FIG 4. Schematic drawing demonstrating the surgical atticotomy margins (dotted line). S indicates scutum; T, tympanic membrane; $M$, malleus.

brane and a remodeled temporal bone without a soft-tissue mass. This is also referred to as "mural cholesteatoma" or "unusual cholesteatoma shell." "3,10,11 Auto-atticotomy can be considered to be the same external decompression process, though less extensive than an auto-mastoidectomy (Fig 3). The autoatticotomy may mimic a surgical atticotomy performed for limited attic cholesteatomas, with removal of the scutum and lower lateral attic wall (Fig 4). The size and shape of an autoatticotomy cavity reflects the size, shape, and location of the original cholesteatoma that produced it. ${ }^{12}$ It is possible that we are seeing a higher incidence of auto-atticotomy compared with auto-mastoidectomy due to detection earlier in the course of the disease because imaging is more easily available.

Pars flaccida cholesteatomas most commonly erode the scutum and lower lateral wall of the attic. Scutal erosion was noted in all patients in our series because this was an inclusion criterion. On MDCT, scutal erosion is typically evaluated in the coronal plane as blunting of the normally sharply pointed bone ridge. However, this erosion can also be appreciated in the axial plane 
where there is widening of the space between the ossicles and the lower lateral attic wall. Smooth lateral expansion of this bony margin can be visualized in a single axial plane image and, in our experience, therefore can occasionally be more noticeable or can support the diagnosis in questionable cases.

Regarding the lateral attic wall, the remodeling of the scutum may be centered either anteriorly, posteriorly, or laterally. ${ }^{11}$ In our series, there was a predilection for anterolateral remodeling, with approximately two-thirds of patients (13/21) demonstrating the greatest widening of the anterolateral measurement (Fig $2 B$ ). Approximately one-fourth of the patients (5/21) demonstrated a posterolateral directionality, with the greatest widening of the posterolateral measurement. A smaller number of patients (2/21) demonstrated scutal erosion in a more straight-lateral fashion, with the greatest widening of the straight-lateral (lateral) measurement. The direct lateral dimension corresponds to the plane of the coronal images and therefore is well-depicted on coronals. The anterolateral and posterolateral measurements lie at a $45^{\circ}$ angle from the coronal plane. In patients with the maximum widening along these axes, the coronal plane might underestimate the extent of attic remodeling. In our study, evaluation of the lateral attic in the coronal plane alone would have underestimated the degree of widening in most of our patients. For this reason, we recommend evaluation on the axial plane as well as the coronal plane.

Nearly half (12/21) of our patients demonstrated auto-atticotomy changes without any CT indication of a residual attic or Prussak space cholesteatoma as evidenced by a soft-tissue component (Fig 3). It is thus especially important to be aware of this imaging appearance, which can alert the clinician to the presence of a previously evacuated cholesteatoma.

For otologists, the dividing line between a deep retraction pocket and a drained cholesteatoma may be difficult to define. It is conceivable that a deep or large retraction pocket can, by itself, create an auto-atticotomy with erosion of the scutum. ${ }^{13}$ A retraction pocket against the long process of the incus has been noted to cause erosion. ${ }^{14}$ So too, a deep retraction pocket could theoretically cause blunting at the scutum without ever forming a collection of keratin or mass. However, a deep retraction pocket seen on clinical examination could also simply represent a previously evacuated cholesteatoma with the outer wall left behind. Once the cholesteatoma evacuates and the keratin reaches the external canal, the "sac" may never obstruct again and there would be no further drainage. The keratin would simply evacuate at the normal rate via the external auditory canal. This process would be appreciated by the otologist as a retraction pocket and may present as a conductive hearing loss without mass or drainage. This differentiation is not crucial because most otologists would treat a deep retraction pocket and an auto-atticotomy from a drained cholesteatoma similarly, depending on the symptoms, including the frequency of drainage and accumulation of debris. $^{15}$

There are other potential or theoretic explanations for loss of the scutum in the absence of soft tissue. The development of the external auditory canal occurs as an invagination of the first branchial cleft, which touches the endoderm of the ascending first branchial pouch to form the tympanic membrane. Mesoderm then grows between the 2 layers, and the mature tympanic membrane is formed of all 3 layers. ${ }^{16}$ Theoretically, the pouch could invaginate further than normal at the superior aspect of the tympanic membrane, but the presence of chronic inflammatory disease in the middle ear and mastoids in almost all of our patients makes this finding unlikely to be a congenital or developmental variant.

\section{CONCLUSIONS}

Evacuated attic cholesteatomas may be difficult to recognize on $\mathrm{CT}$ due to the absence of soft tissue. We propose that widening of the lateral attic and scutal erosion are findings that help to accurately diagnose these patients. We emphasize careful attention to the axial images along the base of the scutum, which can help in recognizing subtle anterior or posterior remodeling of the lateral attic wall and can support questionable findings on coronal images.

Disclosures: Mary E. Cunnane-UNRELATED: Other: WorldCare Clinical, Comments: payment for reviewing radiology studies on patients entered into drug trials for head and neck cancer. Hugh D. Curtin-UNRELATED: Payment for Lectures (including service on Speakers Bureaus): I lecture in Continuing Medical Education but am not sponsored by any company and do not get paid for lecturing; I am not on any Speakers Bureaus, Royalties: none other than books and lecturing; nothing from outside companies related to health care, Travel/Accommodations/Meeting Expenses Unrelated to Activities Listed: If I lecture outside Boston, someone is usually paying meeting expenses and travel (various universities). The International Institute for Continuing Medical Education pays for my travel to a meeting sponsored by Mt. Sinai Hospital.

\section{REFERENCES}

1. Nadol JB, Shuknecht HF. Office examination of the ear. In: Nadol JB, McKenna MJ, eds. Surgery of the Ear and Temporal Bone. 2nd ed: New York: Lippincott Williams \& Wilkins; 2004:5

2. Sadé J. Retraction pockets and attic cholesteatomas. Acta Otorhinolaryngol Belg 1980;34:62-84

3. Swartz JD, Hagiwara M. Inflammatory diseases of the temporal bone. In: Som PM, Curtin HD, eds. Head and Neck Imaging. 5th ed. Philadelphia: Elsevier Mosby; 2011:1207

4. Kikuchi S, Yamasoba T, Iinuma T. An analysis of bone destruction in cholesteatomas by high resolution computed tomography. Auris Nasus Larynx 1993;20:11-17

5. Mafee MF, Kumar A, Yannias DA, et al. Computed tomography of the middle ear in the evaluation of cholesteatomas and other softtissue masses: comparison with pluridirectional tomography. $R a-$ diology 1983;148:465-72

6. Nagar GT. Theories on the origin of attic retraction cholesteatoma. In: Proceedings of the Shambaugh Fifth International Workshop on Microsurgery and Fluctuant Hearing Loss, Huntsville, Alabama. 1977;127-52

7. Swartz JD. The middle ear and mastoid. In: Swartz JD, Loevner LA, eds. Imaging of the Temporal Bone. 4th ed. New York: Thieme; 2009:115

8. Olszewska E, Wagner M, Bernal-Sprekelsen M, et al. Etiopathogenesis of cholesteatoma. Eur Arch Otorhinolaryngol 2004;261:6-24

9. Breinlich T, Giebel W. Histochemical studies of cholesteatoma. Arch Otorhinolaryngol 1984;240:95-101

10. Baráth K, Huber AM, Stampfli P, et al. Neuroradiology of cholesteatomas. AJNR Am J Neuroradiol 2011;32:221-29

11. Nardis PF, Teramo M, Giunta S, et al. Unusual cholesteatoma shell: CT findings. J Comput Assist Tomogr 1988;12:1084-85

12. Vignaud J, Dulac GL, Francois J. Temporal Fosses Nasales, Caviles Accessoires. In: Fischgold H, ed. Traite de Radiodiagnostic. Paris, France: Masson; 1974

13. Sadé J. Pathogenesis of attic cholesteatoma. J Royal Soc Med 1978;71:716-32

14. Borgstein J, Gerritsma TV, Bruce IA. Erosion of the incus in pediatric posterior tympanic membrane retraction pockets without cholesteatoma. Int J Pediatr Otorhinolaryngol 2008;72:1419-23

15. Ars B. Tympanic membrane: retraction pocket. Acta Otorhinolaryngol Belg 1995;49:163-71

16. Curtin HD, Gupta R, Bergeron RT. Embryology, anatomy and imaging of the temporal bone. In: Curtin HD, Som PM, eds. Head and Neck Imaging. 5th ed. Philadelphia: Elsevier Mosby; 2011:1058

AJNR Am J Neuroradiol 35:182-85 Jan 2014 www.ajnr.org 